\title{
Flavour physics with rare, electroweak-penguin, and semileptonic decays at $\mathrm{LHCb}$
}

\section{Zhenzi Wang ${ }^{a, *}$}

${ }^{a}$ Physik-Institut, Universität Zürich,

Winterthurerstrasse 190, Zurich, Switzerland

E-mail: zhenzi.wang@cern.ch

Electroweak-penguin decays are rare, flavour changing neutral current processes that can only take place at loop level in the Standard Model (SM). They provide ideal laboratories for the indirect search of beyond SM effects, as heavy new particles can participate virtually in the decay to cause detectable deviations from theory predictions. The measurements of the properties of these decays have revealed seemingly coherent tensions with the SM. Another group of decays used to test the SM is that of tree-level, semileptonic $b \rightarrow c \ell v$ processes. Due to their large branching fractions and manageable theoretical uncertainties, they are also sensitive probes for new physics effects. These proceedings discuss a selected number of recent measurements made by the $\mathrm{LHCb}$ collaboration in the aforementioned areas.

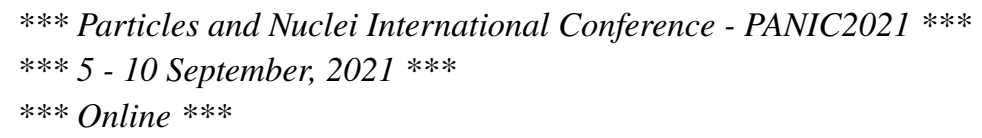

${ }^{*}$ Speaker 


\section{Introduction}

Electroweak-penguin decays featuring the $b \rightarrow s \ell^{+} \ell^{-}$quark transition are flavour changing neutral current (FCNC) processes that can only take place at loop level in the Standard Model (SM). They are sensitive to new physics (NP) effects, which can show up more prominently against the low SM background. $b \rightarrow s \ell^{+} \ell^{-}$processes can be described in a model-independent way using an effective field theory (EFT) formalism, with the Hamiltonian $[1,2]$

$$
\mathcal{H}_{\mathrm{eff}}=-\frac{4 G_{F}}{\sqrt{2}} V_{t b} V_{t s}^{*} \sum_{i=1}^{10} C_{i} O_{i},
$$

where the Wilson coefficients, $C_{i}$, are complex numbers that encode high-energy, short-distance contributions, and $O_{i}$ are the local field operators. NP effects can be incorporated as modifications of Wilson coefficients from their $\mathrm{SM}$ values, i.e. $C_{i}=C_{i}^{\mathrm{SM}}+C_{i}^{\mathrm{NP}}$. In recent years, measurements of observables associated with $b \rightarrow s \ell^{+} \ell^{-}$decays have revealed tensions with SM predictions ${ }^{1}$, which seem to form a consistent pattern when analysed using an EFT approach (e.g. [4]).

Another group of even rarer FCNC processes are the helicity suppressed $B_{(s)}^{0} \rightarrow \ell^{+} \ell^{-}$decays. As their final states are purely leptonic, precise SM predictions of branching fractions can be made. Measurements of these modes are beneficial for the elucidation of the $b \rightarrow s \ell^{+} \ell^{-}$anomalies.

Besides FCNC decays, charged current $b \rightarrow c \ell v$ processes are also promising avenues for NP searches due to their large branching fractions and controllable theoretical uncertainties. They are involved in tests of lepton flavour universality (LFU), some of which hint at deviation from theory predictions.

\subsection{Rare leptonic $B$ decays}

\subsection{1 $B_{(s)}^{0} \rightarrow \mu^{+} \mu^{-}(\gamma)$}

The newest analysis of $B_{(s)}^{0} \rightarrow \mu^{+} \mu^{-}[5,6]$ uses the full set of data collected by the LHCb detector in Run 1 (2011-2012) and Run 2 (2015-2018), corresponding to integrated luminosities of 1,2 and $6 \mathrm{fb}^{-1}$ of proton-proton collisions at center-of-mass energies of 7, 8, and $13 \mathrm{TeV}$, respectively. In addition, the search for the initial state radiation component of the $B_{s}^{0} \rightarrow \mu^{+} \mu^{-} \gamma$ decay is carried out for the first time in the region $m_{\mu \mu}>4.9 \mathrm{GeV} / \mathrm{c}^{2}$ without reconstructing the photon. The branching fractions of all three modes are measured relative to two normalisation modes $\left(B^{+} \rightarrow J / \psi\left(\rightarrow \mu^{+} \mu^{-}\right) K^{+}\right.$and $\left.B^{0} \rightarrow K^{+} \pi^{-}\right)$through an unbinned extended maximumlikelihood fit. The $B_{s}^{0} \rightarrow \mu^{+} \mu^{-}$signal is found to exceed the background-only hypothesis by more than 10 standard deviations $(\sigma)$, while the statistical significance of the $B^{0} \rightarrow \mu^{+} \mu^{-}$and $B_{s}^{0} \rightarrow \mu^{+} \mu^{-} \gamma$ contributions are compatible with the background-only hypothesis. The branching fraction of the $B_{s}^{0}$ mode is measured to be $\mathcal{B}\left(B_{s}^{0} \rightarrow \mu^{+} \mu^{-}\right)=\left(3.09_{-0.43-0.11}^{+0.46+0.15}\right) \times 10^{-9}$, while upper limits of $\mathcal{B}\left(B^{0} \rightarrow \mu^{+} \mu^{-}\right)<2.6 \times 10^{-10}$, and $\mathcal{B}\left(B_{s}^{0} \rightarrow \mu^{+} \mu^{-} \gamma ; m_{\mu \mu}>4.9 \mathrm{GeV} / \mathrm{c}^{2}\right)<2.0 \times 10^{-9}$ are set for the other two modes at $95 \%$ confidence level (CL).

Besides branching fractions, another observable of interest is the effective lifetime, $\tau\left(B_{s}^{0} \rightarrow\right.$ $\mu^{+} \mu^{-}$), which provides a complementary test of the SM. It is extracted by fitting the background subtracted decay time distribution of $B_{S}^{0} \rightarrow \mu^{+} \mu^{-}$candidates, and determined to be $\tau\left(B_{s}^{0} \rightarrow\right.$ $\left.\mu^{+} \mu^{-}\right)=(2.07 \pm 0.29 \pm 0.03) \mathrm{ps}$, which is in agreement with SM prediction.

${ }^{1}$ Please see [3] for a more comprehensive review of the current status. 


\subsection{2 $B_{(s)}^{0} \rightarrow e^{+} e^{-}$}

The decay of $B_{(s)}^{0} \rightarrow e^{+} e^{-}$is strongly suppressed in the SM with branching fractions at the levels of $10^{-15}\left(10^{-13}\right)$, which could be increased to $10^{-10}\left(10^{-8}\right)$ with NP contributions [7]. The first analysis of these channels by the LHCb collaboration [8] uses data obtained in Run 1 and part of Run 2 (up to 2016). No observations are made. As the mass distributions of the $B^{0}$ and $B_{s}^{0}$ modes are highly overlapping due to limited resolution, the branching fraction limit for each is set assuming no contribution from the other. The limits, at $95 \% \mathrm{CL}$, are found to be $\mathcal{B}\left(B_{s}^{0} \rightarrow e^{+} e^{-}\right)<11.2 \times 10^{-9}$ and $\mathcal{B}\left(B^{0} \rightarrow e^{+} e^{-}\right)<3.0 \times 10^{-9}$.

\subsection{Rare $b \rightarrow s \ell^{+} \ell^{-}$decays}

\subsection{1 $B_{s}^{0} \rightarrow \phi\left(\rightarrow K^{+} K^{-}\right) \mu^{+} \mu^{-}$branching fraction}

The latest branching fraction measurement of $B_{s}^{0} \rightarrow \phi\left(\rightarrow K^{+} K^{-}\right) \mu^{+} \mu^{-}$[9] is made using full Run 1 and Run 2 data, in intervals of the dilepton invariant mass squared $\left(q^{2}\right)$. Regions of $q^{2}$ dominated by tree-level $B_{s}^{0}$ decays into final states with a $J / \psi$ or $\psi(2 S)$ meson are vetoed, as is that which is dominated by the decay of $B_{s}^{0} \rightarrow \phi\left(\rightarrow \mu^{+} \mu^{-}\right) \phi$. The differential branching fraction is determined relative to the normalisation mode of $B_{s}^{0} \rightarrow J / \psi \phi$ through an extended maximum likelihood fit. In the $q^{2}$ region of 1.1 to $6.0 \mathrm{GeV}^{2} / \mathrm{c}^{4}$, its value is found to be $(2.88 \pm 0.22) \times$ $10^{-8} \mathrm{GeV}^{-2} \mathrm{c}^{4}$, which lies $3.6 \sigma$ below a SM prediction ${ }^{2}$.

As part of the same analysis, the decay of $B_{s}^{0} \rightarrow f_{2}^{\prime}(1525) \mu^{+} \mu^{-}$is searched for using a similar strategy. This decay is observed for the first time with $9 \sigma$ significance, and its total branching fraction is determined to be $\mathcal{B}\left(B_{s}^{0} \rightarrow f_{2}^{\prime} \mu^{+} \mu^{-}\right)=(1.57 \pm 0.19 \pm 0.06 \pm 0.06 \pm 0.08) \times 10^{-7}$, where the third and fourth uncertainties result from the extrapolation to the full $q^{2}$ range and the branching fraction uncertainty of the normalisation mode, respectively.

\subsection{2 $B^{0} \rightarrow K^{* 0}\left(\rightarrow K^{+} \pi^{-}\right) \mu^{+} \mu^{-}$angular analysis}

The newest angular analysis of $B^{0} \rightarrow K^{* 0}\left(\rightarrow K^{+} \pi^{-}\right) \mu^{+} \mu^{-}$[10] uses data from Run 1 and part of Run 2 (2016 only). The angular distribution of the final state particles of the decay can be described using three angles $\theta_{\ell}, \theta_{K}, \phi$ and $q^{2}$. The differential decay rate, in terms of these variables, is given by [11]

$$
\frac{d^{4} \bar{\Gamma}\left[B^{0} \rightarrow K^{* 0} \mu^{+} \mu^{-}\right]}{d q^{2} d \cos \theta_{\ell} d \cos \theta_{K} d \phi}=\frac{9}{32 \pi} \sum_{i=0}^{9} \bar{I}_{i}\left(q^{2}\right) f_{i}\left(\cos \theta_{\ell}, \cos \theta_{K}, \phi\right),
$$

where $f_{i}$ are combinations of spherical harmonics, and the $I_{i}$ are $q^{2}$ dependent angular coefficients. These can be expressed in terms of the amplitudes of the decay that can be written in terms of the Wilson coefficients $C_{7,9,10}$ and $B \rightarrow K^{*}$ form factors. Different sets of angular observables can be defined using the $I_{i}$ terms, notably the $C P$-averaged, optimised P-basis observables [12], $P_{i}^{(\prime)}$, which are largely form factor independent.

As the triggering, reconstruction and selection of signal candidates distort the angular distributions, corrections are made using functions obtained from simulation that encode this effect. The observable values are extracted from a five dimensional simultaneous fit to the three angles,

${ }^{2}$ Please refer to [9] for additional details. 
$m\left(K^{+} \pi^{-} \mu^{+} \mu^{-}\right)$and $m\left(K^{+} \pi^{-}\right)$of the Run 1 and 2016 samples. They show general consistency with the SM. Tensions are seen in three observables, $F_{L}, A_{F B}$ and $P_{5}^{\prime}$, where local discrepancies in the $q^{2}$ bins of 4.0 to $6.0 \mathrm{GeV}^{2} / \mathrm{c}^{4}$ and 6.0 to $8.0 \mathrm{GeV}^{2} / \mathrm{c}^{4}$ are at levels of $2.5 \sigma$ and $2.9 \sigma$, respectively.

\subsection{3 $B^{+} \rightarrow K^{*+}\left(\rightarrow K_{S}^{0} \pi^{+}\right) \mu^{+} \mu^{-}$angular analysis}

The angular observables of the $B^{+} \rightarrow K^{*+}\left(\rightarrow K_{S}^{0}\left(\rightarrow \pi^{+} \pi^{-}\right) \pi^{+}\right) \mu^{+} \mu^{-}$decay are measured for the first time using full Run 1 and Run 2 data [13]. This decay is the isospin partner of $B^{0} \rightarrow K^{* 0} \mu^{+} \mu^{-}$, and a similar analysis strategy is applied. However, due to comparatively low statistics, a folding technique is used, where sets of transformations of the angles are made to the differential decay rate (same form as Equ. 2) to reduce the number of observables to be determined for a given fit. This improves the stability of the fit without loss of sensitivity. The results are generally in good agreement with the SM. A $3 \sigma$ local discrepancy is seen for the observable $P_{2}$ in the $q^{2}$ interval of 6.0 to $8.0 \mathrm{GeV}^{2} / \mathrm{c}^{4}$. The trend seen in the $P_{5}^{\prime}$ observable shows similarities to what is seen in the analysis of $B^{0} \rightarrow K^{* 0} \mu^{+} \mu^{-}$, although the statistical uncertainties are large.

\subsection{4 $B_{s}^{0} \rightarrow \phi \mu^{+} \mu^{-}$angular analysis}

The newest angular analysis of $B_{s}^{0} \rightarrow \phi \mu^{+} \mu^{-}$[14] is made using data from Run 1 and most of Run 2 (excluding 2015). The analysis strategy used is similar to that of $B^{0} \rightarrow K^{* 0} \mu^{+} \mu^{-}$. However, one important difference is that the final state of this decay is flavour symmetric, which causes some observables such as $P_{5}^{\prime}$ to be inaccessible. Nevertheless, it is interesting to measure the $C P$ asymmetries, which are predicted to be close to zero in the SM but can be large in the presence of NP contributions [15]. The observable values found show good agreement with the SM, with slight tension seen for $F_{L}$ at low $q^{2}$. The $C P$ asymmetries are close to zero.

\subsubsection{Interpretation}

The three most recent angular analyses seems to support what may be a consistent pattern of deviations in $b \rightarrow s \ell^{+} \ell^{-}$decays. This can be seen in the EFT framework. The values of the angular observables obtained from the analyses of the $B^{0} \rightarrow K^{* 0} \mu^{+} \mu^{-}, B^{+} \rightarrow K^{*+} \mu^{+} \mu^{-}$and $B_{s}^{0} \rightarrow \phi \mu^{+} \mu^{-}$ decays are fitted using the Flavio software package [16] varying the real part of the Wilson coefficient $C_{9}$, motivated by global fits to $b \rightarrow s \ell^{+} \ell^{-}$observables (see for example [4]). The results reveal what seems to be a consistent trend, where negative values of $\Delta \operatorname{Re}\left(C_{9}\right)$ are preferred over the SM hypothesis at the level of 2 to $3 \sigma$. However the interpretation of this shift is complicated by SM contributions from charm loop $(c \bar{c})$, which are difficult to reliably calculate (see for example [17]).

\subsection{Semileptonic decays}

Semileptonic decays featuring the $b \rightarrow c \ell v$ transition are involved in several tests of LFU, with some results showing mild tension with the SM. These are carried out by measuring ratios of branching fractions of the form

$$
\mathcal{R}\left(H_{c}\right)=\frac{\mathcal{B}\left(H_{b} \rightarrow H_{c} \tau \nu\right)}{\mathcal{B}\left(H_{b} \rightarrow H_{c} \mu \nu\right)}
$$

where $H_{b}$ and $H_{c}$ refer to hadrons containing $b$ and $c$ quarks, respectively. The usage of ratios allows for the cancellation of some systematic and theoretical uncertainties. The tau is reconstructed through its leptonic (e.g. $\tau^{+} \rightarrow \mu^{+} v_{\mu} \overline{\nu_{\tau}}$ ) or hadronic (e.g. $\tau^{+} \rightarrow \pi^{+} \pi^{-} \pi^{+} \overline{v_{\tau}}$ ) decay modes. 
All analyses of this category are made using Run 1 data only. The most recent measurements include that of $\mathcal{R}\left(D^{*-}\right)$, where the $\tau$ is reconstructed through its hadronic decay [18]. The result of

$$
\mathcal{R}\left(D^{*-}\right) \equiv \frac{\mathcal{B}\left(B^{0} \rightarrow D^{*-} \tau^{+} v_{\tau}\right)}{\mathcal{B}\left(B^{0} \rightarrow D^{*-} \mu^{+} v_{\mu}\right)}=0.291 \pm 0.019 \pm 0.026 \pm 0.013,
$$

is obtained, where the third uncertainty is due to the limited knowledge on the branching fractions of the normalisation mode of $B^{0} \rightarrow D^{*-} \pi^{+} \pi^{-} \pi^{+}$and the $B^{0} \rightarrow D^{*-} \mu^{+} v_{\mu}$ decay.

Another recent result is $\mathcal{R}(J / \psi)$ [19], where the $\tau$ is reconstructed through its hadronic decay mode, and the $J / \psi$ through its decay to two muons. The ratio is found to be

$$
\mathcal{R}(J / \psi) \equiv \frac{\mathcal{B}\left(B_{c}^{+} \rightarrow J / \psi \tau^{+} v_{\tau}\right)}{\mathcal{B}\left(B_{c}^{+} \rightarrow J / \psi \mu^{+} v_{\mu}\right)}=0.71 \pm 0.17 \pm 0.18 .
$$

Both results are consistent with SM predictions (please see [20] and [19] for additional details).

\subsection{Conclusions}

Rare, electroweak-penguin and semileptonic $b \rightarrow c \ell v$ decays provide excellent avenues for the indirect search of new physics. Recent results from the LHCb collaboration in the area of rare leptonic $B$ decays show good agreement with the SM, while existing tensions in observables of $b \rightarrow s \ell^{+} \ell^{-}$decays persist in updates using Run 2 statistics. Updates to LFU testing ratios of semileptonic decays using Run 2 data are expected in the near future. With Run 3 of the LHC on the horizon, and interest from the Belle II collaboration to offer independent insight into the nature of the current anomalies, more interesting results can be expected in the coming years.

\section{References}

[1] W. Altmannshofer and P.t. Ball, JHEP 01 (2009) 019 [0811 . 1214].

[2] A. Ali, G. Giudice and T. Mannel, Z. Phys. C 67 (1993) 417.

[3] N. Watson, Selected Physics Highlights at LHCb, PANIC2021 22nd edition (2021) .

[4] W. Altmannshofer and P. Stangl, Eur. Phys. J. C 81 (2021) 952 [2103 . 13370].

[5] LHCв collaboration 2108.09283.

[6] LHCв collaboration 2108.09284.

[7] R. Fleischer, R. Jaarsma and G. Tetlalmatzi-Xolocotzi, JHEP 05 (2017) 156 [1703 . 10160].

[8] LHCв collaboration, Phys. Rev. Lett. 124 (2020) 211802.

[9] LHCв collaboration, Phys. Rev. Lett. 127 (2021) 151801 [2105 . 14007].

[10] LHCв collaboration, Phys. Rev. Lett. 125 (2020) 011802 [2003. 04831].

[11] U. Egede, T. Hurth, J. Matias, M. Ramon and W. Reece, Journal of High Energy Physics 2008 (2008) 032.

[12] S. Descotes-Genon, T. Hurth, J. Matias and J. Virto, JHEP 05 (2013) 137 [1303 . 5794].

[13] LHСв collaboration, Phys. Rev. Lett. 126 (2021) 161802 [2012 . 13241].

[14] LHCв collaboration, JHEP 11 (2021) 043 [2107. 13428].

[15] C. Bobeth, G. Hiller and G. Piranishvili, JHEP 07 (2008) 106 [0805 . 2525].

[16] D.M. Straub 1810.08132.

[17] A. Khodjamirian, T. Mannel, A.A. Pivovarov and Y.M. Wang, JHEP 09 (2010) 089 [1006. 4945].

[18] LHCв Collaboration collaboration, Phys. Rev. Lett. 120 (2018) 171802.

[19] LHCв Collaboration collaboration, Phys. Rev. Lett. 120 (2018) 121801.

[20] S. Fajfer, J.F. Kamenik and I. Nišandžić, Phys. Rev. D 85 (2012) 094025. 